\title{
SEARCHING FOR THE CONNECTION BETWEEN "LASER AND REPRODUCTION". THOUGHTS AFTER FINISHING THE PROCEEDINGS OF THE THIRD CONGRESS OF THE JAPAN ASSOCIATION FOR LASER REPRODUCTION
}

\author{
Bunpei Ishizuka M.D. \\ Dept. of Gyne. and Obstetrics, St. Marianna Medical University, Japan
}

\begin{abstract}
On March $16^{\text {th }} 2008$, I had the honor of presiding over the third congress for the Japanese Society for Laser Reproduction (JaSLaR) held at Meiji Kinenkan in Tokyo. Laser is the leading edge of photonic \& optic technology and medical applications of lasers are advancing at a phenomenal rate. On the other hand, reproductive medicine is probably the most advancing or evolving field in medicine. Take for example, assisted reproductive technology such as in vitro fertilization. Of all the babies being born, roughly $2 \%$ are the products of pregnancies brought about from this technology. Such advances in reproductive medicine are a blessing for those who hope to bear children in a time and age where the average age of couples getting married for the first time is increasing. However, at the same time these advances pose many strong moral and ethical issues where poor selection may lead to the worst blemish in the history of the human race. In order to avoid such dangers, reproductive medicine is entering an era of increased precision. The application of medical lasers in the field of reproductive medicine can be considered a mean to achieve this, and further developments are welcome.
\end{abstract}

This meeting was the first congress being held by JaSLaR as an fully fledged society. We organized this meeting in hopes of advancing laser reproductive medicine by broadening the scope of lasers and reproductive medicine and thereby searching for connections of the two fields. We especially stressed our interest in blood flow and aging of the ovaries. This is a difficult topic where conventional medicine is failing and where laser medicine shows a possibility. We asked Professor Hirohisa Kurachi from Yamagata University to speak on the topic of decline of female functions related to aging, and asked Dr. Marumo to speak on the decline of male functions. Concerning blood flow and female sexual function, we asked Drs. Ikuko Honda from Yamachika General Hospital, and Norihiro Sugino from Yamaguchi University to speak on the subject. We also planned a workshop "Improvement of ovarian blood flow and increased fecundity" in order to try and elucidate how laser treatment improves reproductive functions through increased blood flow. In the last session "Anti-aging", not only laser rejuvenation but also the recently popular topics concerning dehydroepiandrosterone (DHEA) and melatonin were included.

Approximately 70 people participated in this congress, where both the speakers and participants were able to engage in meaningful discussions. I am confident that we have achieved, if not in whole then at least in part, our primary objectives. I hope for the further development of this society, and I would be extremely proud if contributions from this society help to advance reproductive medicine in Japan.

\section{$3^{\text {rd JaSLaR Program }}$}

\section{Opening Announcement}

President of JaSLaR Bunpei Ishizuka MD

\section{Chairman's Lecture}

The present state of advances in laser surgery and medicine

$$
\begin{array}{ll}
\text { Chair: } & \text { B. Ishizuka } \\
& \text { ( St.Marianna Medical School) }
\end{array}
$$

Speaker: T. Ohshiro

(Japan Medical Laser Laboratory) 


\section{REPORT FROM JASLAR}

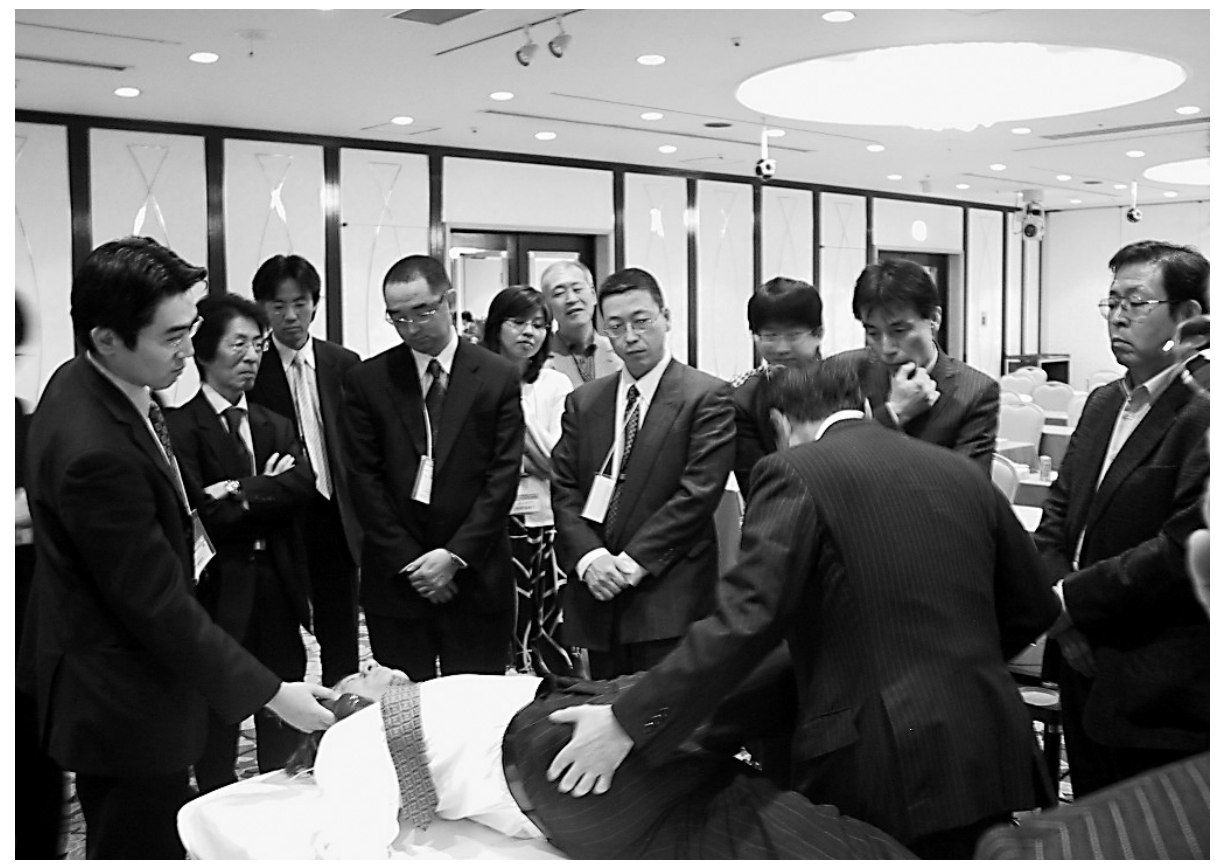

Fig.1 The demonstration "The proximal priority treatment for female infertility using the GaAlAs semi-conductor laser" in Luncheon Seminar.

\section{Educational Lectures}

1. Aging of female functions and healthcare for postmenopausal women

\section{Chair: B. Ishizuka \\ ( St.Marianna Medical School)}

Speaker: H. Kurati (Yamagata University)

2. Male dysfunctions and aging: risk factors and treatment

Chair: H. Ishikawa

(Tokyo Dental and Medical University, IchikawaHospital )

Speaker: K. Marumo

(Tokyo Dental and Medical University, IchikawaHospital )

3. The efficacy of ovarian blood flow evaluation in ART

Chair: Y. Nagata (IVF Nagata clinic)

Speaker: I. Honda

(Yamachika Memorial Hospital)

4. Function and blood flow of female reproductive organs

Chair: M. Inoue (Sannoh Hospital)

Speaker: N. Sugino (Yamaguchi University)
Board Meeting: Japan Society for Laser Reproduction

\section{Luncheon Seminar and Demonstration}

(Co- sponsored by Japan Medical Laser Laboratory)

The proximal priority treatment for female infertility using the GaAlAs semi-conductor laser Speaker: T. Ohshiro Jr. (Ohshiro clinic)

\section{Workshop 1. Laser assisted hatching}

Chair: Y. Araki

(Advanced Reproductive Medicine Laboratory)

1. Comparison between conventional and laser assisted hatching and their efficacy Speaker: A. Fukuda (IVF Osaka clinic)

2. The effect of zona thinning with lasers and its effect on spindle formation

Speaker: H. Yano

(Yano Gyne. \& Obst. IVF center)

3. Reconsiderations on the indications of LAH

Speaker: H. Kamiya (Kamiya Ladies Clinic)

4. The method and record of laser $\mathrm{AH}$ at our clinic Speaker: Y. Asada (Asada Ladies Clinic)

\section{Workshop 2}

Chair: Y. Miyakawa (Ohita University) 
1. The evaluation of ovarian functions through vascular growth factors

Speaker: Y. Kohno (Ohita University)

2. Fecundity and intra-ovarian vascular resistance

Speaker: H. Saitoh

(National Center for Child Health and Development)

3. The efficacy of low reactive level laser therapy for the treatment of female infertility

Speaker: H. Tamura

(Tamura Hideko Gyne. \& Obst. Clinic)

4. A study of blood flow increase by laser treatment and its effect on reproductivity

Speaker: Y. Nagata (IVF Nagata Clinic)

\section{Symposium: Anti-aging}

Chair: S. Suzuki

(Tokyo Reproductive Biology

Symposium)

1. Interaction of laser light and living cells

Speaker: T. Ohshiro Jr. (Ohshiro Clinic)

2. Age changes of the female reproductive organs and genitalia

Speaker: Y. Kurasawa (Ginza Women's Clinic)

3. The application of DHEA for aging ovaries

Speaker: A. Usui (Usui Clinic)

4. Study of HRT performed as part of treatment for cosmesis

Speaker: M. Yoi

(Yoi Clinic/ Dokkyo Medical University)

5. Melatonin and reproduction

Speaker: M. Itoh (St. Marianna Medical University)

\section{Closing Ceremony}

Vice President of JaSLaR Isao Miyagawa MD

\section{from Abstracts}

The Efficacy of Ovarian Blood Flow Evaluation in ART Ikuko Honda, Yamachika Memorial Hospital

In the practice of infertility treatment, ultrasonography (US) gives us a great deal of information concerning the uterus, ovaries and oviducts. In the ovaries, ultrasonography allows us to observe real time changes taking place within them, such as the development of follicles, ovulation and luteinization. Recently attempts have been made not only at evaluating the morphological changes of the follicles and ovaries, but also to assess the function and quality of the follicle and ovum through measurements of ovarian blood flow. In the field of ART, in order to increase the pregnancy rate and to decrease the miscarriage rate, the selection of the most suitable cycle becomes vital. In the past there have been several methods of ultrasonographic evaluation for the collection of better quality ova. The Antral Follicle Count (AFC) is effective in predicting the ovarian response to human menopausal gonadotropin (hMG) stimulation, however such predictions are irrelevant in predicting pregnancies. Follicle monitoring is useful in estimating the day of ovulation, projecting the number of ova collected and predicting ovarian hyperstimulation syndrome (OHSS). However, during hMG stimulation, monitoring each and every follicle for its growth is very time consuming; although the follicular diameter (Wittmack, et al., 1994) is widely used as an index for projecting the number of ova to be collected and fertilization and cleavage of the embryo, this index does not apply to the maturity and quality of a specific ovum. In 1996, Zaidi first reported the correlation between ovarian mesenchymal blood flow and response to stimulation, which powered the conjecture that increased blood flow could result in increased gonadotropin reaching the ovary. In a study by Van Blerkom, et al., the correlation between the pre-collection perifollicular blood flow evaluations by a color Doppler, performed after hCG injection, was reported, in addition to the intra-follicular oxygen concentration, VEGF levels and the incidence of aberrations in chromosome alignment caused by the spindled bodies of $\mathrm{M}$ II stage ova. This put somewhat of a damper on the widely anticipated concept that blood flow evaluation could increase the sensitivity for the selection of the cycle for collection. However, limitations due to the capabilities of the ultrasonography hardware, subjectivity of the examiner and patients' individual idiosyncrasies can affect the results greatly and differences in the results are unavoidable. The most recent blood flow evaluation method by Power Doppler Angiography (PDA) and VOCAL (virtual organ computer-aided analysis) can standardize various parameters of the fields of interest, thereby making objective comparisons possible (Pairleitner et al., 1999). The authors present the characteristics of ovarian blood flow from the point of Vascular Index (VI) and Flow Index (FI) which are the indices of this method. The authors will also present data, collected with this method, on ovarian mesenchymal blood flow at the start of menses and compare it with the perifollicular blood flow just before ova collection, and show how it related to the outcome of the following ART. Perifollicular neovascularization and increased blood flow are essential for the success of ART. The authors discuss the efficacy of ovarian blood flow evaluations and present problems which need to be solved in the future. 